\title{
The Impact of Polyphenol on General Nutrient Metabolism in the Monogastric Gastrointestinal Tract
}

\author{
Mohammed Sharif Swallah $\mathbb{D D}^{1}{ }^{1}$ Hongling Fu, ${ }^{1} \mathrm{He}$ Sun, ${ }^{1}$ Raiffatou Affoh, ${ }^{2}$ and Hansong Yu (iD ${ }^{1}$ \\ ${ }^{1}$ College of Food Science and Engineering, Jilin Agricultural University, Changchun 130118, China \\ ${ }^{2}$ College of Economics and Management, Jilin Agricultural University, Changchun 130118, China
}

Correspondence should be addressed to Mohammed Sharif Swallah; m.s.swallah@gmail.com and Hansong Yu; yuhansong@163.com

Received 24 March 2020; Revised 1 May 2020; Accepted 11 May 2020; Published 10 June 2020

Academic Editor: Quancai Sun

Copyright (c) 2020 Mohammed Sharif Swallah et al. This is an open access article distributed under the Creative Commons Attribution License, which permits unrestricted use, distribution, and reproduction in any medium, provided the original work is properly cited.

\begin{abstract}
Polyphenols are bioactive compounds occurring in plant foods, which are considered significant owing to their contribution to human health and the prevention of chronic diseases. Phenolic compounds mainly depend on plant food structure and the interaction with other food constituents, mostly proteins, lipids, and carbohydrates. The interaction with the food matrices can obstruct or enhance nutrient accessibility and availability and even impair others. Food digestion is a complex process where ingested foods are converted to nutrients via mechanical and enzymatic alterations. The absorption of nutrients predominantly occurs in the small and large intestine, respectively. The metabolised product, however, is the main bioactive component due to their ability to enter the systemic circulation and reach the targeted organs. There is limited knowledge on the cellular uptake, phenolic metabolite, and polyphenolic effect in the gastrointestinal ecosystem. Therefore, improved understanding of the biological properties and stages of dietary phenols is essential for the effective utilization of their therapeutic potentials. This review will explore, summarise, and collate current information on how polyphenols influence nutrient metabolism, bioavailability, and the biotransformation stages.
\end{abstract}

\section{Introduction}

Digestion is an intricate process in which foods ingested are further converted into nutrients through enzymatic and mechanical adjustments, whereby nutrients are further absorbed into the bloodstreams. Naturally, food fragmentation occurs in the mouth and the stomach, whiles nutrients and water absorption, as well as enzymatic digestion predominantly, occur in the large and small intestines [1]. Food composition and antioxidant activity are of great importance in our daily dietary ways owing to their contribution to disease prevention, radical-scavenging [2], and anti-inflammatory activities [3]. Epidemiological research shows that consumption of foods high in bioactive components and antioxidant activities, including phytochemicals, vitamins, and mainly phenolic compounds, such as anthocyanin and carotenoids, has positive merit on human health and could reduce the chances of diseases such as cancer, Alzheimer's, stroke, heart disease, diabetes, and age-related functional decades [4]. Therefore, determination of the phenolic metabolome in the human will not only aid reveal the availability of specific phenolic molecules in the human but also helps to be aware of the relationship between food eaten today and the chances of obtaining certain chronic diseases in the near future [5]. Chronic diseases, including diabetes, cancer, and cardiovascular diseases, are related to the inadequate consumption of plant food. Polyphenols are derived from plants, which include fruits and vegetables, legumes, and plant-based products such as coffee, chocolate, tea, and other beverages. Therefore, it can be tagged as a major constituent of daily human food. On average, the daily consumption of polyphenol in our diet is $1 \mathrm{~g}[6,7]$. Growing evidence and research on polyphenols prove that plant foods play a higher role in the protection of human health and also 
influence several metabolic activities as well as decrease the risk of cardiovascular diseases, among others [7, 8]. Is concluded through several animal studies (in vivo) and in vitro research that polyphenol exhibits oxidative, neuroprotective and chemopreventive activities and anti-inflammatory characteristics, leading to plant food's health protection properties. Hence, it remains vital and necessary to utilize the health merits of these plant foods. In support of this, epidemiological education, the World Health Organization (WHO), and several health organizations encourage the intake of more fruits, vegetables, fibre, and leguminous plants to aid reducing disease risks [9-11]. To further understand the effect of polyphenol on nutrient metabolism and degradation, in vitro digestion models have extensively been used. Still, the greater part of the works has excluded the colonic reactions such as fermentation and others $[12,13]$. This review explores, summarises, and collates the current information on how bioactive compounds (polyphenols) aids in nutrient metabolism as well as its bioavailability.

\section{Food Composition}

2.1. Nutrients and Bioactive Compounds. Apart from the primary known essential nutrients required to ensure that the human body has all it takes for good health, i.e., for the effective functioning of the central nervous and immune system, as well as disease prevention, [14]. There are other natural and synthetic compounds present in our daily diets that as well interfere with food digestion, enzyme activities, and the absorption of nutrients. They are termed antinutrients and include the following: protease inhibitors, amylase inhibitors, and lipase inhibitors. [15]. Bioactive compounds are molecules that present therapeutic potential with an influence on oxidative stress, metabolic disorders, energy intake, and reducing proinflammatory state [4]. Recent studies acknowledge the need for nonnutrients (bioactive), which are supposed to be of health benefits. Bioactive compounds are regarded to be different from nutrients because it is not essential for life compared with macro- or micronutrients but have an effect on the body as a whole or specific cells and tissues. It is believed that such compounds from both animal and plant sources have a positive impact on human health. These compounds include non-pro-vitamin A carotenoids, polyphenols, peptides, phytosterols, and fatty acids. Bioactive compounds act as antioxidants and further provide the defence that prevents disease spread and enhance body repairs [15-20].

2.2. Phenolic Profiles. Phenols can as well be called phenolics, which are natural compounds occurring in plant foods such as cereals, coffee, fruits, vegetables, and wine. Chemically, polyphenols are class of aromatic compounds characterised by hydroxyl groups attached to an aromatic hydrocarbon group. Thus, polyphenol is a term explaining natural products having not less than double phenyl rings bearing single or additional hydroxyl substituents, while phenols are used in expressing a phenyl ring bearing single or extra hydroxyl substituents (i.e., phenol is in a monomeric form while polyphenols are in the polymeric form). Based on their substituting groups and the number of phenolic rings, polyphenols are categorised into nonflavonoids, phenolic acids, and flavonoids. Generally, phenols have double or more hydroxyl groups, which are the biologically active substance that occurs in food plants mostly consumed by a considerable number of people [21-24]. Polyphenols constitute most subclasses of the active biological phytochemicals, including flavonoids (e.g., isoflavones and anthocyanins), nonflavonoids (e.g., tannins, stilbenes, and lignans) and phenolic acids (e.g., hydroxybenzoic acids and hydroxycinnamic acids) [25]. Flavonoids are the most studied phenolic groups, with over 9000 identified different structures in nature. It forms a major heterogeneous subgroup with a variety of compounds bearing similar diphenyl propane skeleton (C6-C3-C6). Sequentially, it is further subclassified due to their structural variation, including proanthocyanins, dihydroflavonols, flavonols, flavan-3-ols or flavanols, anthocyanidins, isoflavones, flavanones, and flavones [26-28] Table 1 presents the classes of polyphenols and chemical structures as well as their respective known food sources and compounds [25, 29-32].

\subsection{Bioavailability and Bioaccessibility of Bioactive Com-} pounds (Polyphenol) within the GI Tract (Gastrointestinal Tract). Bioactive compounds with diverse chemical structures affect bioavailability and biological properties, while the antinutritional factor can as well decrease or inhibit digestion enzymes [33]. Therefore, to get a detailed understanding of absorption and metabolism of phenols within the GI tract, it is better to consider the chemical structure of a phenol, since different chemical structures affect their redox potential. Comparably, polyphenols with double close hydroxyl groups are better and have free radical scavenging ability to those with a single hydroxyl group per [34]. Bioactive compounds can alter metabolic processes and give out positive functions such as inhibition of receptor activities, induction of enzymes, antioxidant effect, and inhibition of gene expression [35]. The changes in bacterial microbiota in the gastrointestinal tract are associated with metabolic disorders including, diabetes, obesity, or nonalcoholic fatty liver disease [36]. The in vitro test is repeatedly used for the determination of almost all biological activities, using polyphenol in their natural states, i.e., as it is in food without any breakdown. However, polyphenols are extensively metabolised both in tissues and by the colonic microflora [6]. Bioavailability of polyphenols within the gastrointestinal tract depends on the phenolic secondary microbial metabolites acting within the colons. Therefore, it is vital to explore the metabolites as well as biological properties and activities. A current finding reveals that there was a good correlation between in vivo and in vitro bioavailability of the same compound under the concentration-time curve. Therefore, cocultures can be used to copycat absorption in an in vivo test [13]. The bioaccessibility of a polyphenol is solely dependent on the bioavailability of the food matrix after successful absorption into the bloodstream [37]. For that reason, bioavailability can be defined as the proportion of bioactive compounds that are successfully absorbed into the bloodstream 
TABLE 1: Detailed classification of natural polyphenols.

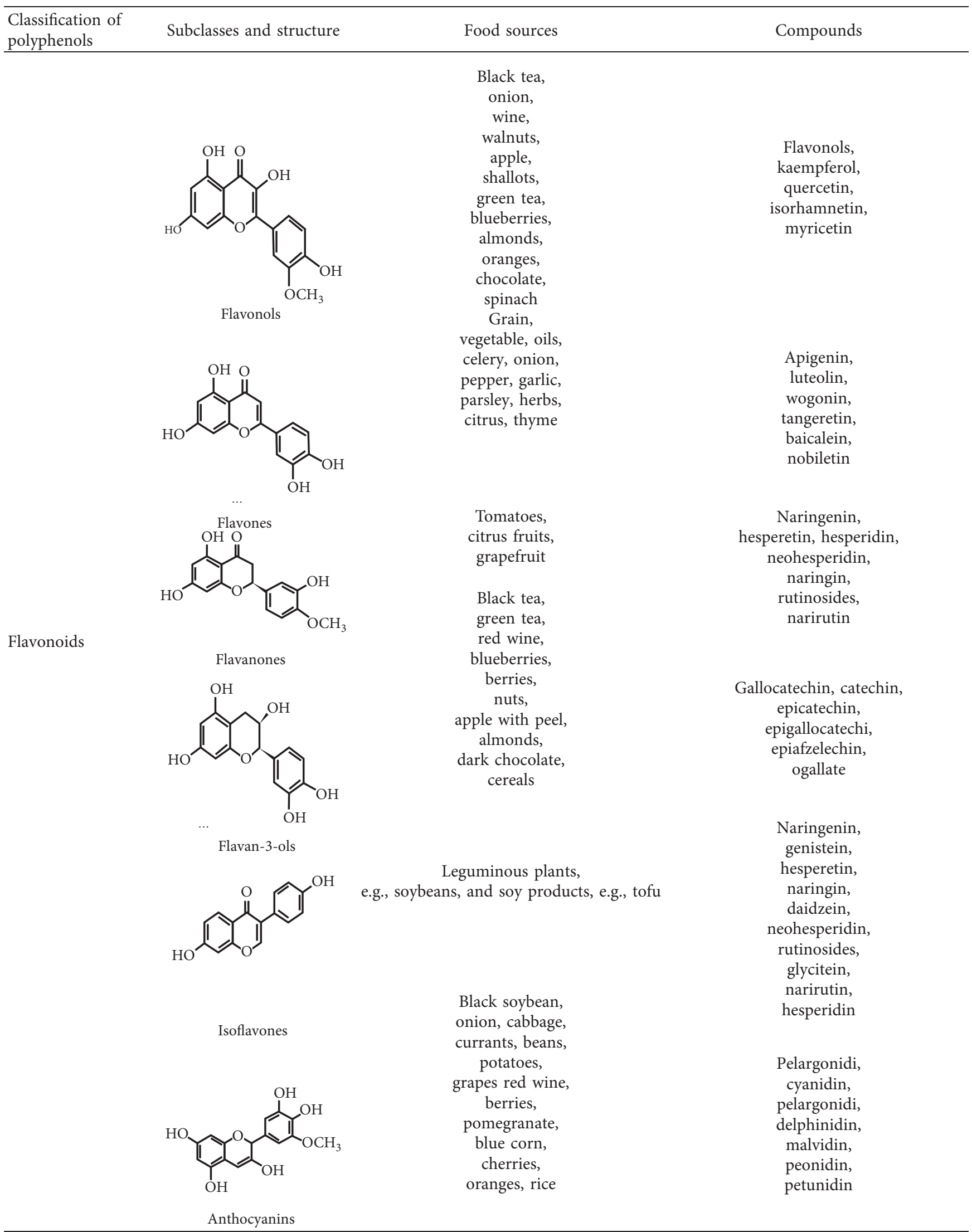


TABLE 1: Continued.

$\begin{aligned} & \text { Classification of } \\ & \text { polyphenols }\end{aligned}$
Phenolic acids<smiles>Oc1ccc(C=Cc2cc(O)cc(O)c2)cc1</smiles>

Stilbenes<smiles>COc1cc(CC(CO)C(CO)Cc2ccc(O)c(OC)c2)ccc1O</smiles>

Lignans<smiles>OC1Cc2ccccc2OC1c1ccccc1</smiles>

Red wine, grape fruits

Resveratrol

Cereals,

flaxseed,

linseed,

algae,

pumpkin,

potato,

leguminous crops,

cereals,

vegetables, fruits

Leguminous plant, grape

Gallotannins (gallic acid), Ellagitannins

(ellagic acid, punicalin, and punicalagin)

Tannin

for metabolic utilization [38]. However, bioaccessibility can as well be defined as the fraction of bioactive compounds released from the food matrix after digestion and becoming available for intestinal absorption [39]. In this case, the antioxidants will only be readily available for absorption after successful digestion. Before absorption, the majority of dietary polyphenols are metabolised by the colonic microbiota, and a small proportion will be directly absorbed in the upper gastrointestinal tract [40]. Hence, the microbial metabolism is a prerequisite for absorption. Again, gut bacteria can modulate polyphenols via several mechanical means including demethylation, decarboxylation, hydrolysis, reduction, and ring-cleavage as well as modulate the biological activities of a compound. Dietary polyphenols systematically depend on the synergistic action that polyphenols may exert after entering circulation, endogenous factors, and other dietary constituents [41, 42]. Several factors interfere with the absorption and release of polyphenols, and the difficulty in nutrient absorption within the intestine is due to the changes in the interaction of other compounds, chemical nutrients from the food, suppressors in the food composition, and overall metabolism. Several studies aimed at defining the bioavailability after digestion, since the effectiveness of all polyphenols or antioxidants in vivo depends on the concentration of the food matrix as well as both bioaccessibility and bioavailability [43-45]. Figure 1 presents a summary of the bioavailability and bioaccessibility determination methods [46].

\section{Role of Polyphenol in the Gastrointestinal Tract}

The gastrointestinal tract (GIT) is the host for over one thousand different microbial species, which include both 


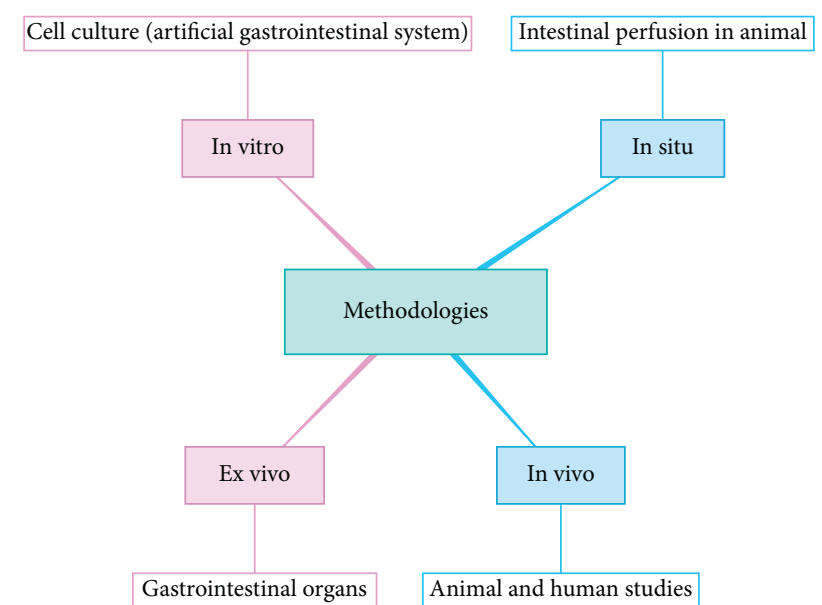

Figure 1: Bioavailability and bioaccessibility determination methods of bioactive compounds.

biotic and symbiotic bacterial species. At present, it is estimated that 500-1000 species hinder the GIT from attaining the highest concentration in the colon. Yet, out of these bacterial species (e.g., Eubacterium, actinobacteria, Escherichia coli, Proteobacteria, Lactobacillus, Bifidobacterium, Bacteroidetes, Bacteroides, and Firmicutes), few are known to aid in the phenolic metabolic activities $[47,48]$. Though the modulation and formation of bioactive polyphenol-derived metabolites, and the colonic microbiota might both merit the host cells through nutrient processing, boosting of the immune system, and gastrointestinal protection; the mechanism is yet to be defined $[49,50]$. The natural process of digestion begins in the mouth by chewing and emitting of bioactive compounds from food substance. For successful bioaccessibility of nutrients during gastrointestinal digestion, the hydrolytic enzymes, gastric acids, the physicochemical properties of the secretion act on the food bolus, resulting in the stabilisation of the phenolic components as well as facilitating the release of compounds from the food matrix $[51,52]$. The human body recognises the absorbed phenolic components as a xenobiotic substance, and their absorption is quite low compared with macro- and micronutrients and thereby has to metabolise it to increase their bioavailability rate. Its metabolism starts from the upper intestinal epithelia and proceeds to the lower intestine, the liver, and then to the peripheral tissues, which include adipose tissue and the kidneys. To facilitate the excretion of urine, the hepatic enzymes convert the molecules by increasing or reducing hydroxyl groups (first phase) and inflecting them to other molecules (second phase), thereby maximising their water solubility. Cytochrome P450 monooxygenases are one of the key enzyme systems that aid in metabolising xenobiotic substances $[53,54]$. The human gastrointestinal system begins digestion with the simulation of gastric acids (pepsin-HCL), followed by the breakdown of food macromolecules within the small intestine with the aid of bile and pancreatic juice and finally with dialysis [55]. Figure 2 presents the main mechanisms within the human digestive system [46].
3.1. Biotransformation Process of Polyphenol within the Mouth, Stomach, Small Intestine, and Colon. There is limited knowledge concerning the cellular uptake of phenolic metabolites in the gastrointestinal tract due to the complex processes in their absorption [56]. The route of phenolic absorption can either be via the stomach and small intestine or possibly absorbed by the colon after chemical modification by the colonic microbiota. During the transformation process, dietary polyphenols under conjugation and catabolic reactions change and get absorbed into the bloodstream and thereafter get excreted either in the urine or via the bile. The substrate or unabsorbed is passed out into faeces [57]. Recent research shows that out of $100 \%$ total polyphenolic intake, only $5-10 \%$ will be absorbed within the small intestine (i.e., monomeric, dimeric structure, and aglycones can be absorbed in the small intestine) and the 90-95\% will be in the large intestine lumen together with other conjugates excreted by the bile. They are then exposed to the intestinal enzymes and gut microbiota. Therefore, the colonic microbiota is in charge of further breaking down of the polyphenolic structures into smaller molecules to aid easy absorption $[58,59]$. The incorporation of polyphenol is variable within the digestive tract. Phenolic concentration in the food matrix does not affect the bioavailability rate but relatively depends on the functional group present and the chemical structure of the dietary polyphenols. Besides, they may be other biologically active molecules present in the body, which can as well influence the absorption and metabolic rate as well as its rapid elimination to reach the blood circulation and the targeted organs [60-62]. Naturally, polyphenols in the human body are in glycoside, ester, and polymeric forms that need to be hydrolysed to facilitate rapid absorption by either gut microbiota or intestinal enzymes. With the aid of $\beta$-glucosidase during the oral cavity salivation process, hydrolysation is likely to begin in the mouth though not thoroughly researched. Once absorbed into the small intestine, phenolic compounds with less complex structures undergoes extensive phase I biotransformation (oxidation, reduction, or hydrolysis) and converts into water-soluble metabolite in the enterocytes before reaching the liver. The complex phenolic compounds not absorbed in the small intestine reach the colon. Once in the large intestine, glycosides are hydrolysed by the gut microbiota resulting in the formation of aglycones through opening the heterocycle. This catabolic action decreases the complex structure of the phenolic hydroxyl group into lowmolecular-weight phenolic metabolites that can be absorbed. When absorbed, the molecules reach the liver via the hepatocytes, where they are again subjected to phase II biotransformation process (conjugation) which includes methylation, glucuronidation, and sulfation or sulfate derivatives. The conjugation stage enhances easy absorption (hydrophobicity) of the molecules and aids in rapid elimination. The metabolites then enter the systemic circulation where they are distributed to the targeted organs or eliminated in the urine $[58,63]$. Figure 3 shows the metabolic route for dietary polyphenol in the human gastrointestinal tract. 


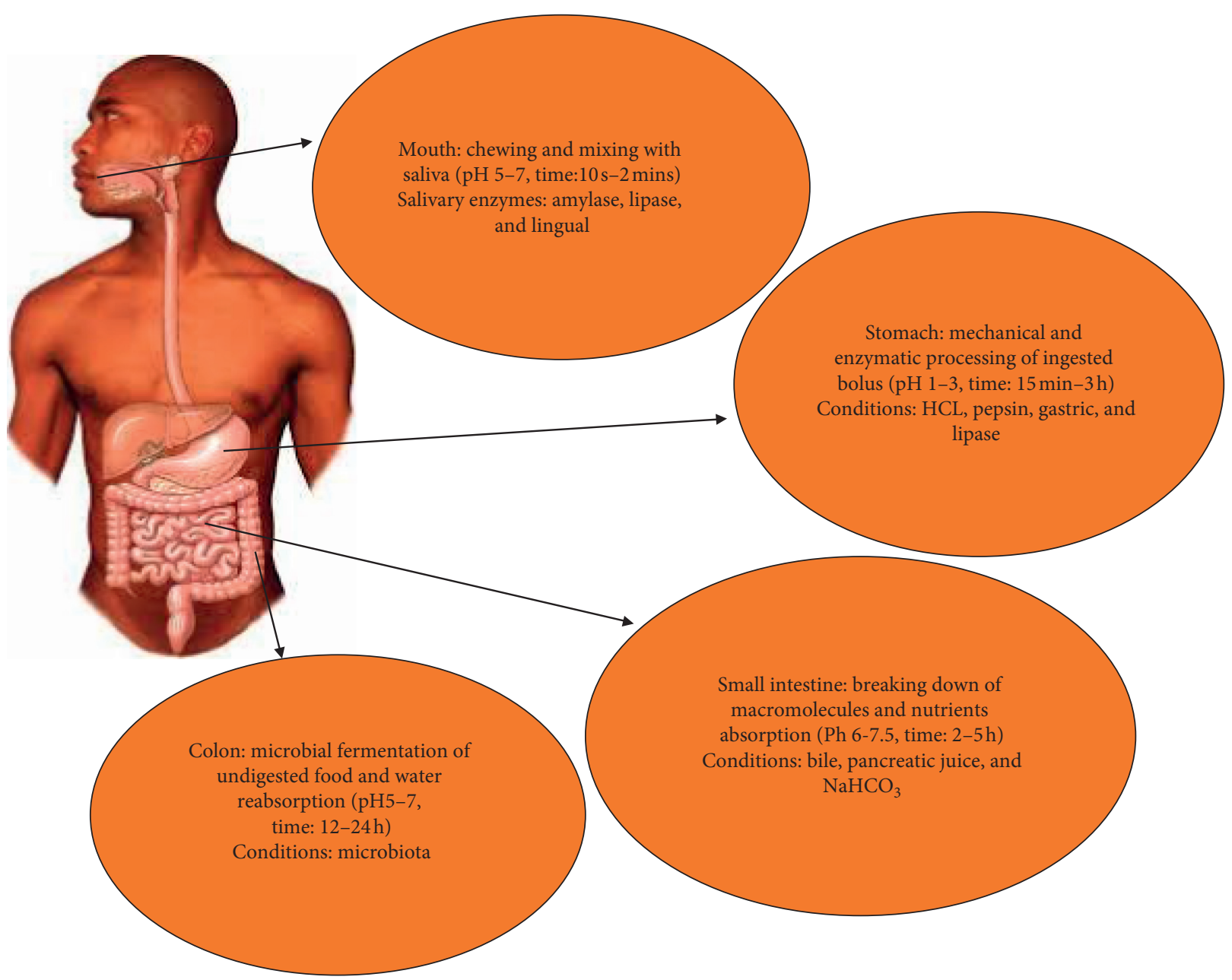

FIgUre 2: The main mechanisms of the human digestive system.

3.2. Influence of Polyphenol on Gut Microbiota. Recent studies show the connection between polyphenol and the intestinal microbiota and how parallel rich phenolic-foods can influence the activity and composition of the microbiota. Polyphenol intake improves the health effects of the gastrointestinal microbiota by activating the intestinal immune function, SCFA (short-chain fatty acids) excretion, and other physiological processes [32]. Though the chemistry and biotransformation process of polyphenols in the digestive tract is well defined [64], little is known of the effect polyphenol possesses on the gut's ecosystem. Recent advances confirm that the rate of bacterial growth in the guts ecosystem is influenced by the dose and structure of the polyphenol in the diet as well as the bacteria's species or strain [65]. The larger composition of bacteria is known to produce high resistance to polyphenolic influence than fewer bacteria composition due to the differences in cell structures [66]. However, it is impossible to predict the required dosage that will yield a beneficial or consequential effect in vivo [57]. The antibacterial effect is influenced by several factors, including cell wall damaging [67], permeability of cell wall [68], or production of $\mathrm{H}_{2} \mathrm{O}_{2}$ [69]. The microbiota colonizing the gastrointestinal tract of human is mainly in charge of modulating the immune system [70] and also contributes to the production of several vitamins including vitamins $K$ and $B$. It can as well contribute to a negative health effect. Atypical microflora action can influence the growth of neoplasm in the gut due to mutagen and carcinogen production (Bacteroides, E. coli, and faecalis) [71]. In Frejnagel and Juskiewicz's study [72] on the effect of green tea, blue-berried honeysuckle and chokeberry's phenolic extract on rat caecal fermentation process were assessed. The outcome of the studies indicates that the addition of the three-extract resulted in an increment in weight of the gut wall as compared with the control animals. However, only rat-fed diet supplemented with green tea extract polyphenol yielded a significant $(P<0.05)$ increment in gut-weight content. Also, the experiment showed a decrease in $\mathrm{pH}$ and concentration of ammonia in rat fed with honeysuckle-supplemented polyphenolic extracts. But Leverat et al. [73] used 1\% Quebracho tannins to supplement the diet of a rat, and it recorded an increase in the rat's digester $\mathrm{pH}$. Two-way variance analysis confirmed there is a dose-dependent decrement in comparison with the control animals. On the contrary, honeysuckle extract in rat diets elevated the enzyme activity of the caeca while chokeberry extract in rat diets recorded a dose-dependent reduction in all enzyme-tested activities. It was only green tea extract in 


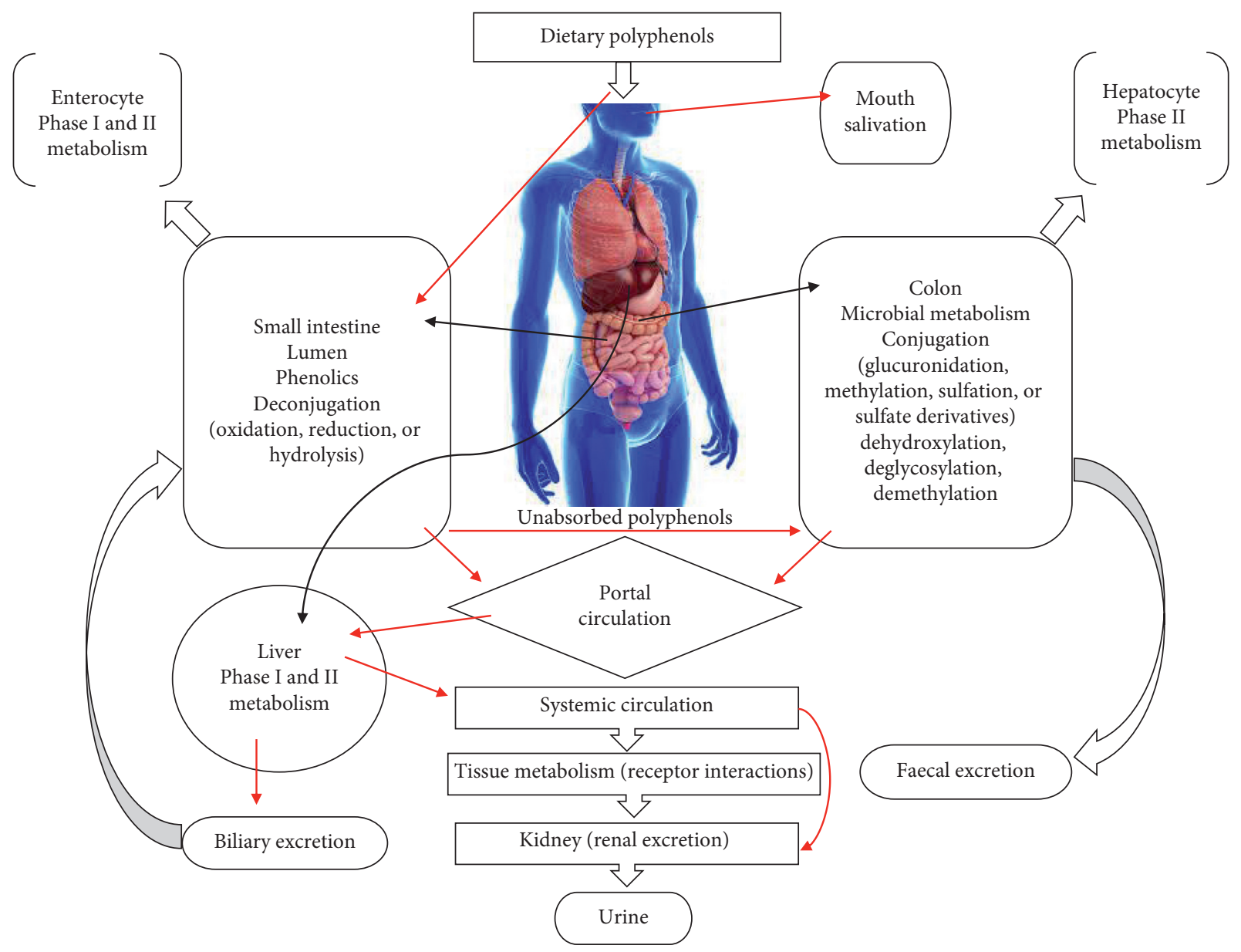

FIGURE 3: Metabolic route for polyphenol in the human gastrointestinal tract.

rat diets that resulted in a significant $(P<0.05)$ inhibition of enzymatic activity. The results indicated that supplementation of diets with phenolic extracts could physically and biochemically affect caecal parameters in rats [72]. Also, research by Smith et al. on bacterial mechanisms in overcoming the inhibitory effect of dietary tannins reveals that rats fed with tannin-rich diet had a significant increase in Bacteroides groups with a decrease clostridium leptum cluster [74]. The research by Tzonuis et al. [75], on flavonol monometer-induced changes to the human faecal microflora, was also reviewed. The study shows that (+) catechin significantly inhibits the growth rate of Clostridium histolyticum and improves the growth rate of E. coli and members of the Clostridium coccoides-Eubacterium rectale group, while the growth rate of Lactobacillus spp. and Bifidobacterium remains relatively unaltered. Varricchio et al. [76] also analysed the influence of polyphenols from olive mill wastewater on the intestine, alveolar macrophages, and blood leukocytes of pigs. They found an increase in the number of lamina propria and intraepithelial leukocytes present in the treated animal's gastrointestinal tracts in contrast with the control group. Immunopositive cells were found mainly in the infiltrate leukocytes in the intraepithelial leukocytes from the gastrointestinal tracts of the controls, with zero amount of immunopositive cells present in the treated animal. The study carried out by Puupponen-Pimia et al. [77], on the antimicrobial effect of phenolic compounds from berries, reveals that lactic acid bacteria were more resistant to polyphenol compounds except for myricetin, which inhibited the growth rate of all lactic acid bacteria with no effect on salmonella. The extract exhibited a growth-inhibiting effect on gram-negative bacteria. The results indicated that the synergistic action of polyphenols is the cause of the inhibition. This proves that gut microbiota plays a significant role in both nutrient and polyphenols metabolism and can as well influence their interactions.

3.3. Influence of Polyphenol on Nutrient Metabolism. Polyphenols are biologically active compounds that solely depend on plant foods structure and interactions with other food constituents, mainly the macronutrients (proteins, lipids, and carbohydrates). This interaction with food matrices can affect or enhance phenolic accessibility and availability and even impair others [78]. A substantial amount of dietary proteins, carbohydrates, and all fats are absorbed before reaching the large intestine. Few human types of research have explored the influence of polyphenols on bacterial metabolism of macronutrients as they are highly absorbed in the early stage of the gut, and modification 
observed seems to reflect on the balance between these digestible macronutrients [79].

3.3.1. Influence on Protein. The influence of phenolic compounds on the activities of protease and protein substrate accessibility determines the digestibility of protein. These are due to the binding of phenolic compounds onto the endogenous protein, which includes the gastric and intestinal mucus, digestive enzymes, and protein saliva. which are the main factors influencing protein digestibility and metabolism. It can as well hinder both the catalytic site and the substrate-binding site, which will end up reducing proteolytic activities. Phenolic compounds can influence protein hydrolysis via the interaction with various protein substrates and protease. The binding activity of phenolic compounds is linked with the binding affinity to enzymes since enzymes are as well protein. Therefore, their molecular size, structure, and composition of an amino acid are all determined by the enzymatic affinity to polyphenols. Generally, the bulkiness and structure of a phenol mainly influence the structure of the protein by either stabilising or loosening the structure, which, in a way, affects enzyme activity [80]. The protein type also affects microbiota composition, which, on the other hand, influences phenolic activities [81]. Data from human and animal studies on phenolic and microbiota influence on dietary protein were thoroughly reviewed. A result of studying the effect of dietary protein source on rat metabolic effect has been evaluated [81]. The effect of $20 \%$ protein from casein, soy, and fish was evaluated in rat diets for 16 weeks. The authors reported a difference in the caecal short-chain fatty acids (SCFA); that is, the fish protein diet recorded an increase in the total SCFA and lactic acid, which was twice that of soy and casein protein diets. The concentration of butyrate was also greater in animals fed with soy diet in contrast with the other two groups. Caecal indole concentration was twice higher with the soy and fish diets as compared with casein diets. Ammonia was higher in the fish diet compared with other diets. Phenol and $\mathrm{H}_{2} \mathrm{~S}$ were as well higher with fish and soy diets against the casein diets. The result from in vivo studies with rats revealed that the digestion of whey proteins could be obstructed by the reactions with some phenolic compounds, mainly chlorogenic acids [82]. The authors focused on determining whether chlorogenic acid can influence the nutritional quality of $\beta$-lactoglobulin and protein digestibility. The results showed a decrease in nitrogen digestibility on higher derivatization with chlorogenic acid, in contrast with zero or lower derivatization at all. It was concluded that good nutritional quality of whey proteins could only be threatened at high-level derivatization with chlorogenic acids. In addition to this, rat fed with soy protein derivatives displayed an increase in urinary and faecal nitrogen and true nitrogen digestibility, and the total utilization of protein was affected. The protein digestibilitycorrected amino acid score (PDCAAS) for tryptophan, lysine, and sulfur-containing amino acids were all decreased [83]. Therefore, the reduction in protein digestibility as an impact of covalent interaction or binding with polyphenol plays a role in the decreasing of polyphenol released from food matrices during digestion, thereby reducing bioavailability. Also, the binding to protein phenolic compounds is protected from adverse stomach condition [80].

3.3.2. Influence on Carbohydrates. Carbohydrate is made up of amylose, which is a linear $\alpha$-1,4-linked glucose polymer, and a highly branched amylopectin consisting of a linear $\alpha$-1,4-linked glucose chain with $\alpha$-1,6-linked branch chains. Pancreatic and salivary $\alpha$-amylases catalyse the endo-hydrolysis of $\alpha$-1,4-glucosidic linkages releasing mainly maltose, maltotriose, and related $\alpha$-1,6-oligomers. In addition, the main enzymes responsible for the breakdown of carbohydrates into glucose are $\alpha$-amylase and $\alpha$-glucosidase [8]. The metabolism of carbohydrates begins in the oral cavity and continues in the pancreas and the small intestine. Any disturbance in metabolism can yield health concerns, including diabetes, obesity, and even dental caries [78]. The majority of carbohydrate ingested is entirely digested in the small intestine; a small proportion escapes and reaches the colon. They are known as resistant starch (RS), it is subclassified into four forms of starch, i.e., chemically modified starch, physically inaccessible starch, retrograded starch, and certain granular starch that are resistant to enzyme digestion. The RS not only does inhibit metabolism but can as well provide the colonic microbiota with the largest single-source dietary-derived energy [79]. Besides drugs, polyphenols can as well aid in the inhibition of carbohydrate's metabolic enzyme activity. The structure of polyphenol can be disadvantageous to enzymatic actions, especially via the hydroxylation process. That is, during the carbon atom hydroxylation process, at some point in rings, it can inhibit enzymatic activities and the glycosylation of the hydroxyl group can as well increase enzyme activities [78]. There have been several in vivo and in vitro studies on the impact of polyphenol on carbohydrates metabolism as well as glucose homeostasis. A study indicated that phenolic extract from chokeberry could serve as an effective $\alpha$-glucosidase inhibitor, which will subsequently decrease the blood glucose level and provide protection against diabetes. Therefore, continuous consumption of chokeberry could aid in the reduction of blood glucose in mild hypercholesterolemia patients and as well decrease the levels of glycated haemoglobin of noninsulin-dependent diabetes patients $[84,85]$. Hence, phenolic compounds could act as a potential antidiabetic agent. A series of animal experiments have shown inhibition of polyphenols to $\alpha$-amylase and $\alpha$-glucosidase activities. The inhibitory phenols include catechins, isoflavones, anthocyanins, flavonols, flavanones, flavones, ellagitannins, and proanthocyanins [8]. The study carried by Adisakwattana et al. [86] analysed $\alpha$-amylase, $\alpha$-glucosidase, and protein glycation inhibitory activities of edible plants. It was observed that hypoglycaemic effects of tannins extracted from grape seed could originate not only from the inhibition of digestive enzymes but can as well be obtained from the inhibition of the glycation process. Again, a human study involving the consumption of coffee with a high carbohydrate or high-fat meal and nondairy creamier was observed 
to impede the absorption of gut microbiota catabolites $[87,88]$. A phenolic (resveratrol) rat experiment has been suggested to increase glucose uptake through the enhancement of estrogenic receptor- $\alpha$, which in turn promotes Glut 4 expression via the AKT pathway and phosphatidyl inositol-3 kinase (PI3K) [89]. Finally, Yamashita's [90] study on the comparison between low- and high-degree of polymerization procyanidin and of antihyperglycemic activities was also reviewed. And, it was observed that procyanidins with a high-degree polymerisation have a strong influence on $\alpha$-glucosidase inhibition as compared with less polymerised ones. Therefore, an antihyperglycemic impact on procyanidins with a small degree of polymerization could be attributed to the stimulation of glucose uptake by glucose transporter in the skeletal muscle. Though phenolic compounds can inhibit the performance of carbohydrate digestive enzymes ( $\alpha$-glucosidase and $\alpha$-amylase), yielding to a delay in digestion, it can as well modulate glucose uptake after consumption of carbohydrate-rich meals.

3.3.3. Influence on Lipids. Compared with other macronutrients like protein-polyphenol interactions, lipids interactions with polyphenol are not well known and have less attention, with the exception of plant-derived oil, i.e., mainly olive oil, which has recently gained attention owing to its best source of oleic fatty acids as well as total monounsaturated fatty acids (MUFA) [78]. Polyphenol is a known antioxidant soluble to water that moves through the portal veins and lipids as well have less influence on the absorption of hydrophilic molecules (polyphenols). Other authors suggested that bioavailability and absorption of anthocyanins from the gastrointestinal might be hindered by the presence of lipids, sugar, and carbohydrates in the whole blueberry extract [91]. Mullen et al.'s [92] study on the metabolism of strawberries with and without cream in humans was reviewed. The study confirmed a delay in digestion of anthocyanins from strawberries when they were consumed with cream, but its bioavailability was not altered. The study conducted on the effect of curcumin on adipogenesis in 3T3-L1 adipocytes and angiogenesis and obesity in C57/BL mice was reviewed. It was observed via the activation of AMPK (adenosine monophosphate-activated protein kinase) that curcumin impeded the synthesis of glycerol lipids by hindering the activity of (GPAT-1) glycerol-3-phosphate acyl transferase-1, which in turn esterifies fatty acids into glycerol to form triglycerides for storage [93]. Peng et al. as well affirmed in recent research that vitexin, i.e., a flavonoid glycoside (an apigenin-8-C-glucoside), activated AMPK $\alpha$, which is a key enzyme in controlling and reducing fat accumulation [94-96]. Other animal and cell culture studies revealed that the addition of a phenolic compound (resveratrol) to rat isolated hepatocytes turns to hinder the synthesis of triglycerides and fatty acid, i.e., through the lowering effect of lipids. Again, it lowers adipogenesis in human isolated adipocytes and as well encourages lipolytic activity in adipocytes via the induction of cAMP [97]. Finally, a study that evaluated the effect of polyphenol-rich diet on the composition postprandial lipoprotein in individuals at high cardiometabolic risk was reviewed, and the study revealed a decreased level of cholesterol and triglycerides. The authors concluded that polyphenol-rich diet reduces the postprandial lipid content of very-low-density lipoproteins (VLDL) and as well modifies the composition of low-density lipoproteins (LDL) particles- which in turn became richer in triglycerides and of which high-density lipoproteins (HDL) became triglyceride poor [98]. Therefore, polyphenolic interaction in the cell membranes can cause changes to the lipid profile.

\section{Conclusions}

Polyphenols are biologically active compounds that are wellthought-out as one of the essential antinutritional factors. The reviewed animal, human, and cell culture studies present the influence of the interactions between polyphenols and nutrients in the monogastric gastrointestinal tract as well as their consequence after metabolism. The interactions can reflect on the metabolism and absorption of interacting compounds, which depends on the food matrices and its biotransformation by the component of the gastrointestinal microbiota. Though the metabolism of polyphenol is considered to aid in the protection of other food compounds and maintain the digestive health via the stimulation and modulation of microbial balance, it can as well impact the organoleptic properties of the food matrices either positively or negatively depending on the phenolic dosage. Again, the complexity of phenolic biotransformation and the association between food matrices and the intestinal microbiome is incompletely understood. Therefore, future researches are essential to investigate the safety required phenolic dosage and the catabolic reactions of polyphenols and conduct a well-designed and controlled human trial, as well as assess the microbial activities at all stages of biotransformation of polyphenol compounds and the impact on human health in order to understand completely.

\section{Data Availability}

No data were used to support this study, and all references were duly acknowledged.

\section{Conflicts of Interest}

The authors declare no conflicts of interest.

\section{Acknowledgments}

The authors would like to thank the Earmarked Fund for Modern Agro-Industry Technology Research System (CARS-04) for the support.

\section{References}

[1] A. Guerra, L. Etienne-Mesmin, V. Livrelli, S. Denis, S. Blanquet-Diot, and M. Alric, "Relevance and challenges in modeling human gastric and small intestinal digestion," Trends in Biotechnology, vol. 30, no. 11, pp. 591-600, 2012. 
[2] C. Pereira, L. Barros, A. M. Carvalho, and I. C. F. R. Ferreira, "Nutritional composition and bioactive properties of commonly consumed wild greens: potential sources for new trends in modern diets," Food Research International, vol. 44, no. 9, pp. 2634-2640, 2011.

[3] S. Cicerale, L. Lucas, and R. Keast, "Biological activities of phenolic compounds present in virgin olive oil," International Journal of Molecular Sciences, vol. 11, no. 2, pp. 458-479, 2010.

[4] N. Siriwardhana, N. S. Kalupahana, M. Cekanova, M. LeMieux, B. Greer, and N. Moustaid-Moussa, "Modulation of adipose tissue inflammation by bioactive food compounds," The Journal of Nutritional Biochemistry, vol. 24, no. 4, pp. 613-623, 2013.

[5] J. Mursu, J. K. Virtanen, T.-P. Tuomainen, T. Nurmi, and S. Voutilainen, "Intake of fruit, berries, and vegetables and risk of type 2 diabetes in Finnish men: the kuopio ischaemic heart disease risk factor study," The American Journal of Clinical Nutrition, vol. 99, no. 2, pp. 328-333, 2014.

[6] A. Scalbert, C. Morand, C. Manach, and C. Rémésy, "Absorption and metabolism of polyphenols in the gut and impact on health," Biomedicine \& Pharmacotherapy, vol. 56, no. 6, pp. 276-282, 2002.

[7] L. Hooper, P. A. Kroon, E. B. Rimm et al., "Flavonoids, flavonoid-rich foods, and cardiovascular risk: a meta-analysis of randomized controlled trials," The American Journal of Clinical Nutrition, vol. 88, no. 1, pp. 38-50, 2008.

[8] K. Hanhineva, R. Törrönen, I. Bondia-Pons et al., "Impact of dietary polyphenols on carbohydrate metabolism," International Journal of Molecular Sciences, vol. 11, no. 4, pp. 1365-1402, 2010.

[9] C. Mertz, A.-L. Gancel, Z. Gunata et al., "Phenolic compounds, carotenoids and antioxidant activity of three tropical fruits," Journal of Food Composition and Analysis, vol. 22, no. 5, pp. 381-387, 2009.

[10] L. G. Espinosa-Alonso, A. Lygin, J. M. Widholm, M. E. Valverde, and O. Paredes-Lopez, "Polyphenols in wild and weedy Mexican common beans (Phaseolus vulgaris L.)," Journal of Agricultural and Food Chemistry, vol. 54, no. 12, pp. 4436-4444, 2006.

[11] W. Diet, "Nutrition and the prevention of chronic diseases. Report of a joint WHO/FAO expert consultation," vol. 916Technical Report Series, pp. 34-38, WHO, Geneva, Switzerland, 2003.

[12] T. Bohn, G. J. McDougall, A. Alegría et al., "Mind the gapdeficits in our knowledge of aspects impacting the bioavailability of phytochemicals and their metabolites-a position paper focusing on carotenoids and polyphenols," Molecular Nutrition \& Food Research, vol. 59, no. 7, pp. 1307-1323, 2015.

[13] S. Sadeghi Ekbatan, M. Iskandar, L. Sleno et al., "Absorption and metabolism of phenolics from digests of polyphenol-rich potato extracts using the Caco-2/HepG2 co-culture system," Foods, vol. 7, no. 1, p. 8, 2018.

[14] H. H. Vorster and M. J. Gibney, "Food and nutrition-related diseases: the global challenge," in Introduction to Human Nutrition, Wiley, Hoboken, NJ, USA, 2009.

[15] Y. Birk, "The Bowman-Birk inhibitor. Trypsin-and chymotrypsin-inhibitor from soybeans," International Journal of Peptide and Protein Research, vol. 25, no. 2, pp. 113-131, 2009.

[16] B. Halliwell and J. M. Gutteridge, Free Radicals in Biology and Medicine, Oxford University Press, Oxford, UK, 2015.

[17] J. Fernstom and M. Fernstom, "Nutrition and the brain," in Nutrition and Metabolism. The Nutrition Society Textbook, S. A. Lanham-New, I. A. Macdonald, and H. M. Roche, Eds., pp. 155-184, Wiley-Blackwell, Chichester, UK, 2nd edition, 2011.
[18] D. Graf, R. D. Cagno, F. Fåk et al., "Contribution of diet to the composition of the human gut microbiota," Microbial Ecology in Health and Disease, vol. 26, p. 26164, 2015.

[19] S. Astley and P. Finglas, "Nutrition and health," in Reference Module in Food Science, Elsevier, Amsterdam, The Netherlands, 2016.

[20] M. S. Swallah, H. Sun, R. Affoh, H. Fu, and H. Yu, "Antioxidant potential overviews of secondary metabolites (polyphenols) in fruits," International Journal of Food Science, vol. 2020, Article ID 9081686, 8 pages.

[21] N. Balasundram, K. Sundram, and S. Samman, "Phenolic compounds in plants and agri-industrial by-products: antioxidant activity, occurrence, and potential uses," Food Chemistry, vol. 99, no. 1, pp. 191-203, 2006.

[22] J. B. Harborne, "General procedures and measurement of total phenolics," Methods in Plant Biochemistry, vol. 1, pp. 1-28, 1989.

[23] R. Puupponen-Pimiä, A.-M. Aura, K.-M. Oksman-Caldentey et al., "Development of functional ingredients for gut health," Trends in Food Science \& Technology, vol. 13, no. 1, pp. 3-11, 2002.

[24] G. Pereira-Caro, B. Fernández-Quirós, I. A. Ludwig, I. Pradas, A. Crozier, and J. M. Moreno-Rojas, "Catabolism of citrus flavanones by the probiotics Bifidobacterium longum and Lactobacillus rhamnosus," European Journal of Nutrition, vol. 57, no. 1, pp. 231-242, 2018.

[25] L. Basheer and Z. Kerem, "Interactions between CYP3A4 and dietary polyphenols," Oxidative Medicine and Cellular Longevity, vol. 2015, Article ID 854015, 15 pages, 2015.

[26] M. P. Corcoran, D. L. McKay, and J. B. Blumberg, "Flavonoid basics: chemistry, sources, mechanisms of action, and safety," Journal of Nutrition in Gerontology and Geriatrics, vol. 31, no. 3, pp. 176-189, 2012.

[27] M. Bingham, "The metabolism of polyphenols by the human gut microbiota," in Gastrointestinal Microbiology, pp. 155168, Springer Science \& Business Media, Berlin, Germany, 2006.

[28] C. Andrés-Lacueva, A. Medina-Remon, R. Llorach et al., "Phenolic compounds: chemistry and occurrence in fruits and vegetables," in Fruit and Vegetable Phytochemicals, Wiley Online Library, Hoboken, NJ, USA, 2010.

[29] C. G. Fraga, K. D. Croft, D. O. Kennedy, and F. A. TomásBarberán, "The effects of polyphenols and other bioactives on human health," Food \& Function, vol. 10, no. 2, pp. 514-528, 2019.

[30] K. Liu, M. Luo, and S. Wei, "The bioprotective effects of polyphenols on metabolic syndrome against oxidative stress: evidences and perspectives," Oxidative Medicine and Cellular Longevity, vol. 2019, Article ID 6713194, 16 pages, 2019.

[31] M. Wiciński, J. Gębalski, E. Mazurek et al., "The influence of polyphenol compounds on human gastrointestinal tract microbiota," Nutrients, vol. 12, no. 2, p. 350, 2020.

[32] K. Kawabata, Y. Yoshioka, and J. Terao, "Role of intestinal microbiota in the bioavailability and physiological functions of dietary polyphenols," Molecules, vol. 24, no. 2, p. 370, 2019.

[33] A. Septembre-Malaterre, F. Remize, and P. Poucheret, "Fruits and vegetables, as a source of nutritional compounds and phytochemicals: changes in bioactive compounds during lactic fermentation," Food Research International, vol. 104, pp. 86-99, 2018.

[34] S. Jovanovic, S. Steenken, M. G. Simic, Y. Hara, S. V. Jovanovic, and M. G. Simic, "Antioxidant properties of flavonoids: reduction potentials and electron transfer reactions of flavonoid radicals," Flavonoids in Health and Disease, pp. 137-161, 1998. 
[35] J. M. Carbonell-Capella, M. Buniowska, F. J. Barba, M. J. Esteve, and A. Frígola, "Analytical methods for determining bioavailability and bioaccessibility of bioactive compounds from fruits and vegetables: a review," Comprehensive Reviews in Food Science and Food Safety, vol. 13, no. 2, pp. 155-171, 2014.

[36] B. Zhu, X. Wang, and L. Li, "Human gut microbiome: the second genome of human body," Protein \& Cell, vol. 1, no. 8, pp. 718-725, 2010.

[37] D. Tagliazucchi, E. Verzelloni, D. Bertolini, and A. Conte, "In vitro bio-accessibility and antioxidant activity of grape polyphenols," Food Chemistry, vol. 120, no. 2, pp. 599-606, 2010.

[38] H. Palafox-Carlos, J. F. Ayala-Zavala, and G. A. GonzálezAguilar, "The role of dietary fiber in the bioaccessibility and bioavailability of fruit and vegetable antioxidants," Journal of Food Science, vol. 76, no. 1, pp. R6-R15, 2011.

[39] C. Manach, G. Williamson, C. Morand, A. Scalbert, and C. Rémésy, "Bioavailability and bioefficacy of polyphenols in humans. I. Review of 97 bioavailability studies," The American Journal of Clinical Nutrition, vol. 81, no. 1, pp. 230S-242S, 2005.

[40] M. V. Selma, J. C. Espín, and F. A. Tomás-Barberán, "Interaction between phenolics and gut microbiota: role in human health," Journal of Agricultural and Food Chemistry, vol. 57, no. 15, pp. 6485-6501, 2009.

[41] M. A. Lila, "From beans to berries and beyond: teamwork between plant chemicals for protection of optimal human health," Annals of the New York Academy of Sciences, vol. 1114, no. 1, pp. 372-380, 2007.

[42] R. H. Liu, "Health benefits of fruit and vegetables are from additive and synergistic combinations of phytochemicals," The American Journal of Clinical Nutrition, vol. 78, no. 3, pp. 517S-520S, 2003.

[43] J. Pérez-Jiménez, J. Serrano, M. Tabernero et al., "Bioavailability of phenolic antioxidants associated with dietary fiber: plasma antioxidant capacity after acute and long-term intake in humans," Plant Foods for Human Nutrition, vol. 64, no. 2, pp. 102-107, 2009.

[44] J. Parada and J. M. Aguilera, "Food microstructure affects the bioavailability of several nutrients," Journal of Food Science, vol. 72, no. 2, pp. R21-R32, 2007.

[45] D. I. Santos, J. M. A. Saraiva, and A. A. Vicente, Margarida Moldão-Martins Linking Landscape, Environment, Agriculture and Food (LEAF), School of Agriculture, University of Lisbon, Lisbon, Portugal, 2019.

[46] D. I. Santos, "Methods for determining bioavailability and bioaccessibility of bioactive compounds and nutrients," in Innovative Thermal and Non-Thermal Processing, Bioaccessibility and Bioavailability of Nutrients and Bioactive Compounds, pp. 23-54, Elsevier, Amsterdam, The Netherlands, 2019.

[47] M. Kutschera, W. Engst, M. Blaut, and A. Braune, "Isolation of catechin-converting human intestinal bacteria," Journal of Applied Microbiology, vol. 111, no. 1, pp. 165-175, 2011.

[48] S. Bibbò, G. Ianiro, V. Giorgio et al., "The role of diet on gut microbiota composition," European Review for Medical and Pharmacological Sciences, vol. 20, no. 20, pp. 4742-4749, 2016.

[49] B. Vitali, M. Ndagijimana, F. Cruciani et al., "Impact of a synbiotic food on the gut microbial ecology and metabolic profiles," BMC Microbiology, vol. 10, no. 1, p. 4, 2010.

[50] C. Duggan, J. Gannon, and W. A. Walker, "Protective nutrients and functional foods for the gastrointestinal tract," The American Journal of Clinical Nutrition, vol. 75, no. 5, pp. 789-808, 2002.
[51] D. Tagliazucchi, E. Verzelloni, and A. Conte, "The first tract of alimentary canal as an extractor. Release of phytochemicals from solid food matrices during simulated digestion," Journal of Food Biochemistry, vol. 36, no. 5, pp. 555-568, 2012.

[52] A. Chandrasekara and F. Shahidi, "Bioaccessibility and antioxidant potential of millet grain phenolics as affected by simulated in vitro digestion and microbial fermentation," Journal of Functional Foods, vol. 4, no. 1, pp. 226-237, 2012.

[53] A. Bento-Silva, V. M. Koistinen, P. Mena et al., "Factors affecting intake, metabolism and health benefits of phenolic acids: do we understand individual variability?" European Journal of Nutrition, vol. 59, no. 4, pp. 1275-1293, 2020.

[54] M. M. Appeldoorn, J.-P. Vincken, H. Gruppen, and P. C. H. Hollman, "Procyanidin dimers A1, A2, and B2 are absorbed without conjugation or methylation from the small intestine of rats," The Journal of Nutrition, vol. 139, no. 8, pp. 1469-1473, 2009.

[55] M. Bermudezsoto, F. Tomasbarberan, and M. Garciaconesa, "Stability of polyphenols in chokeberry (Aronia melanocarpa) subjected to in vitro gastric and pancreatic digestion," Food Chemistry, vol. 102, no. 3, pp. 865-874, 2007.

[56] A.-M. Aura, "Microbial metabolism of dietary phenolic compounds in the colon," Phytochemistry Reviews, vol. 7, no. 3, pp. 407-429, 2008.

[57] G. Williamson and M. N. Clifford, "Role of the small intestine, colon and microbiota in determining the metabolic fate of polyphenols," Biochemical Pharmacology, vol. 139, pp. 24-39, 2017.

[58] F. Cardona, C. Andrés-Lacueva, S. Tulipani, F. J. Tinahones, and M. I. Queipo-Ortuño, "Benefits of polyphenols on gut microbiota and implications in human health," The Journal of Nutritional Biochemistry, vol. 24, no. 8, pp. 1415-1422, 2013.

[59] J. L. Donovan, V. Crespy, C. Manach et al., "Catechin is metabolized by both the small intestine and liver of rats," The Journal of Nutrition, vol. 131, no. 6, pp. 1753-1757, 2001.

[60] G. Annunziata, M. Maisto, C. Schisano et al., "Colon bioaccessibility and antioxidant activity of white, green and black tea polyphenols extract after in vitro simulated gastrointestinal digestion," Nutrients, vol. 10, no. 11, p. 1711, 2018.

[61] A. Crozier, D. Del Rio, and M. N. Clifford, "Bioavailability of dietary flavonoids and phenolic compounds," Molecular Aspects of Medicine, vol. 31, no. 6, pp. 446-467, 2010.

[62] M. D. Archivio, "Polyphenols, dietary sources and bioavailability," Annali-Istituto Superiore di Sanita, vol. 43, no. 4, pp. 348-361, 2007.

[63] L. Lavefve, L. R. Howard, and F. Carbonero, "Berry polyphenols metabolism and impact on human gut microbiota and health," Food \& Function, vol. 11, no. 1, pp. 45-65, 2020.

[64] J. F. Stevens and C. S. Maier, "The chemistry of gut microbial metabolism of polyphenols," Phytochemistry Reviews, vol. 15, no. 3, pp. 425-444, 2016.

[65] D. Hervert-Hernández and I. Goñi, "Dietary polyphenols and human gut microbiota: a review," Food Reviews International, vol. 27, no. 2, pp. 154-169, 2011.

[66] R. Puupponen-Pimia and L. Nohynek, S. Hartmann-Schmidlin, Berry phenolics selectively inhibit the growth of intestinal pathogens," Journal of Applied Microbiology, vol. 98, no. 4, pp. 991-1000, 2005.

[67] R. A. Kemperman, S. Bolca, L. C. Roger, and E. E. Vaughan, "Novel approaches for analysing gut microbes and dietary polyphenols: challenges and opportunities," Microbiology, vol. 156, no. 11, pp. 3224-3231, 2010.

[68] Y. Xie, J. Chen, A. Xiao, and L. Liu, "Antibacterial activity of polyphenols: structure-activity relationship and influence of 
hyperglycemic condition,” Molecules, vol. 22, no. 11, p. 1913, 2017.

[69] V. Sant'Anna and E. Biondo, E. M. Kolchinski, Total polyphenols, antioxidant, antimicrobial and allelopathic activities of spend coffee ground aqueous extract," Waste and Biomass Valorization, vol. 8, no. 2, pp. 439-442, 2017.

[70] L. V. Hooper, D. R. Littman, and A. J. Macpherson, "Interactions between the microbiota and the immune system," Science, vol. 336, no. 6086, pp. 1268-1273, 2012.

[71] P. Mandal, "Molecular mechanistic pathway of colorectal carcinogenesis associated with intestinal microbiota," $A n$ aerobe, vol. 49, pp. 63-70, 2018.

[72] S. Frejnagel and J. Juskiewicz, "Dose-dependent effects of polyphenolic extracts from green tea, blue-berried honeysuckle, and chokeberry on rat caecal fermentation processes," Planta Medica, vol. 77, no. 9, pp. 888-893, 2011.

[73] M. A. Leverat, O. Texier, F. Regerat, C. Demigne, and C. Remesey, "Comparison of the effect of condensed tannin and pactin on caecal fermentations and lipid metabolism in rat," Nutrition Research, vol. 13, no. 4, pp. 427-433, 1993.

[74] A. H. Smith, E. Zoetendal, and R. I. Mackie, "Bacterial mechanisms to overcome inhibitory effects of dietary tannins," Microbial Ecology, vol. 50, no. 2, pp. 197-205, 2005.

[75] X. Tzounis, J. Vulevic, V. Livrelli, G. G. C. Kuhnle et al., "Flavanol monomer-induced changes to the human faecal microflora," British Journal of Nutrition, vol. 99, no. 4, pp. 782-792, 2008.

[76] E. Varricchio, E. Coccia, G. Orso et al., "Influence of polyphenols from olive mill wastewater on the gastrointestinal tract, alveolar macrophages and blood leukocytes of pigs," Italian Journal of Animal Science, vol. 18, no. 1, pp. 574-586, 2019.

[77] R. Puupponen-Pimiä, L. Nohynek, C. Meier et al., "Antimicrobial properties of phenolic compounds from berries," Journal of Applied Microbiology, vol. 90, no. 4, pp. 494-507, 2001.

[78] N. Kardum and M. Glibetic, "Polyphenols and their interactions with other dietary compounds: implications for human health," in Advances in Food and Nutrition Research, pp. 103-144, Elsevier, Amsterdam, The Netherlands, 2018.

[79] C. Shortt, O. Hasselwander, A. Meynier et al., "Systematic review of the effects of the intestinal microbiota on selected nutrients and non-nutrients," European Journal of Nutrition, vol. 57, no. 1, pp. 25-49, 2018.

[80] T. D. C. Velickovic and D. J. Stanic-Vucinic, "The role of dietary phenolic compounds in protein digestion and processing technologies to improve their antinutritive properties," Comprehensive Reviews in Food Science and Food Safety, vol. 17, no. 1, pp. 82-103, 2018.

[81] C. An, T. Kuda, T. Yazaki, H. Takahashi, and B. Kimura, "Caecal fermentation, putrefaction and microbiotas in rats fed milk casein, soy protein or fish meal," Applied Microbiology and Biotechnology, vol. 98, no. 6, pp. 2779-2787, 2014.

[82] K. J. Petzke, S. Schuppe, S. Rohn, H. M. Rawel, and J. Kroll, "Chlorogenic acid moderately decreases the quality of whey proteins in rats," Journal of Agricultural and Food Chemistry, vol. 53, no. 9, pp. 3714-3720, 2005.

[83] S. Rohn, K. J. Petzke, H. M. Rawel, and J. Kroll, "Reactions of chlorogenic acid and quercetin with a soy protein isolate-influence on the in vivo food protein quality in rats," Molecular Nutrition \& Food Research, vol. 50, no. 8, pp. 696-704, 2006.

[84] M. Bräunlich, R. Slimestad, H. Wangensteen, C. Brede, K. Malterud, and H. Barsett, "Extracts, anthocyanins and procyanidins from Aronia melanocarpa as radical scavengers and enzyme inhibitors," Nutrients, vol. 5, no. 3, pp. 663-678, 2013.

[85] A. Skoczyñska, I. Jedrychowska, R. Poręba et al., "Influence of chokeberry juice on arterial blood pressure and lipid parameters in men with mild hypercholesterolemia," Pharmacological Reports, vol. 59, no. 1, pp. 177-182, 2007.

[86] S. Adisakwattana, P. Jiphimai, P. Prutanopajai, B. Chanathong, S. Sapwarobol, and T. Ariyapitipan, "Evaluation of $\alpha$-glucosidase, $\alpha$-amylase and protein glycation inhibitory activities of edible plants," International Journal of Food Sciences and Nutrition, vol. 61, no. 3, pp. 295-305, 2010.

[87] M. Renouf, C. Marmet, P. Guy et al., "Nondairy creamer, but not milk, delays the appearance of coffee phenolic acid equivalents in human plasma," The Journal of Nutrition, vol. 140 , no. 2, pp. 259-263, 2010.

[88] D. Scherbl, M. Renouf, C. Marmet et al., "Breakfast consumption induces retarded release of chlorogenic acid metabolites in humans," European Food Research and Technology, vol. 243, no. 5, pp. 791-806, 2017.

[89] J. Ahn, I. Cho, S. Kim, D. Kwon, and T. Ha, "Dietary resveratrol alters lipid metabolism-related gene expression of mice on an atherogenic diet," Journal of Hepatology, vol. 49, no. 6, pp. 1019-1028, 2008.

[90] Y. Yamashita, "Comparison of anti-hyperglycemic activities between low-and high-degree of polymerization procyanidin fractions from cacao liquor extract," Journal of Food and Drug Analysis, vol. 20, pp. 283-287, 2012.

[91] P. Li, M. Lu, M. T. A. Nguyen et al., "Functional heterogeneity of CD11c-positive adipose tissue macrophages in diet-induced obese mice," Journal of Biological Chemistry, vol. 285, no. 20, pp. 15333-15345, 2010.

[92] W. Mullen, C. A. Edwards, M. Serafini, and A. Crozier, "Bioavailability of pelargonidin-3-O-glucoside and its metabolites in humans following the ingestion of strawberries with and without cream," Journal of Agricultural and Food Chemistry, vol. 56, no. 3, pp. 713-719, 2008.

[93] A. Ejaz, D. Wu, P. Kwan, and M. Meydani, "Curcumin inhibits adipogenesis in 3T3-L1 adipocytes and angiogenesis and obesity in C57/BL mice," The Journal of Nutrition, vol. 139, no. 5, pp. 919-925, 2009.

[94] Y. Peng, Q. Sun, W. Xu et al., "Vitexin ameliorates high fat diet-induced obesity in male C57BL/6J mice via the AMPK $\alpha$ mediated pathway," Food \& Function, vol. 10, no. 4, pp. 1940-1947, 2019.

[95] Q. Sun, J. Lin, Y. Peng, R. Gao, and Y. Peng, "Flubendiamide enhances adipogenesis and inhibits AMPK $\alpha$ in 3T3-L1 adipocytes," Molecules, vol. 23, no. 11, p. 2950, 2018.

[96] Y. Peng, R. Gan, H. Li et al., "Absorption, metabolism, and bioactivity of vitexin: recent advances in understanding the efficacy of an important nutraceutical," Critical Reviews in Food Science and Nutrition, pp. 1-16, 2020.

[97] M. Meydani and S. T. Hasan, "Dietary polyphenols and obesity," Nutrients, vol. 2, no. 7, pp. 737-751, 2010.

[98] G. Della Pepa, C. Vetrani, M. Vitale et al., "Effects of a diet naturally rich in polyphenols on lipid composition of postprandial lipoproteins in high cardiometabolic risk individuals: an ancillary analysis of a randomized controlled trial," European Journal of Clinical Nutrition, vol. 74, no. 1, pp. 183192, 2020. 